\section{Incentivos y desincentivos de la industria farmacéutica privada para la I+D de nuevos medicamentos}

\author{
Incentives and disincentives for research and \\ development of new drugs by the pharmaceutical \\ industry
}

\section{${ }_{1}^{1}$ Departamento de Ciencias Económicas y Administrativas, Universidad Simón Bolívar, Caracas, Venezuela. \\ Correspondencia P. C. Curcio \\ Departamento de Ciencias Económicas y Administrativas, Universidad Simón Bolívar. Valle de Sartenejas, Edificio de Estudios Generales, Departamento de Ciencias Económicas y Sociales, piso 1o, oficina 34 , Caracas, Venezuela. pcurcio@usb.ve}

\begin{abstract}
The authors present a model with factors that influence research and development decisions by the pharmaceutical industry: risk of disease transmission and possibility of control; casefatality and the presence of cure or treatments; income; number of persons who demand the medicine; and opportunity costs for the company. Companies tend to invest in markets with inelastic demand (highly contagious diseases with no possibility of controlling transmission and/or very lethal diseases without treatment) and/or where there is a large population or high per capita income. Companies tend not to invest in markets where marginal costs exceed marginal income, particularly when costs increase permanently as a consequence of rising opportunity costs generated by foregoing profit in other markets. In such cases, policies to subsidize $R \& D$ are not effective, and policies must be orientated towards strengthening basic and applied research by public institutions.
\end{abstract}

Pharmaceutical Industry; Health Policies; Pharmaceutical Preparations; Motivation
Pasqualina Curcio Curcio 1

\section{Introducción}

Todos los años se enferman y mueren miles de personas a causa de enfermedades tropicales, a pesar de esta situación, los recursos invertidos por parte de la industria farmacéutica para la investigación y el desarrollo (I+D) de medicamentos para combatirlas son muy bajos. En los últimos 25 años sólo se han desarrollado 15 nuevos medicamentos para estas enfermedades que representan el $12 \%$ de la carga de morbilidad mundial, versus 179 nuevos medicamentos nuevos que fueron desarrollados para enfermedades cardiovasculares que representan el $11 \%$ de la carga total de enfermedades 1 .

La principal diferencia entre los dos tipos de enfermedades radica en que las primeras afectan primordialmente a los países en desarrollo de bajos ingresos, y no afectan a los países de altos ingresos, mientras que las otras representan la mayor carga de morbilidad de los países desarrollados y con altos ingresos. Investigaciones realizadas por el Comité de los Derechos de Propiedad Intelectual de la Organización Mundial de la Salud muestran que los mecanismos de incentivos que otorgan los derechos de propiedad intelectual no son suficientes para investigar en aquellas enfermedades cuyos mercados son los de países de bajos ingresos 2. La industria farmacéutica orientará sus investigaciones hacia aquellas enfermedades que afectan a los países desarrollados y con mayor capacidad de pago. 
No obstante, existe una serie de interrogantes a los cuales es imposible dar respuesta si consideramos la capacidad de pago como único factor determinante del comportamiento de la industria farmacéutica en el momento de tomar las decisiones de invertir en I+D. ¿Por qué aunque sólo se han reportado 291 casos de la gripe aviar y 172 muertes desde el 2003 hasta el 11 de abril de 2007, la cual se ha manifestado en países en desarrollo, la industria farmacéutica ha orientado recursos para la I+D y ya existen más de 40 ensayos clínicos de vacunas contra la gripe pandémica en seres humanos?

¿Por qué si la epidemia de la diabetes afecta a 246 millones de personas en el mundo, la mayoría habitantes con altos niveles de ingreso, y si cada año 7 millones de personas desarrollan la enfermedad y 3 millones mueren por causas relacionadas ${ }^{3}$, no se han invertido suficientes recursos para desarrollar una cura 3 ?

La dificultad que se nos presenta al tratar de responder estas interrogantes nos lleva a plantearnos la hipótesis de que no es sólo el nivel de ingreso de los países, y por consiguiente su capacidad de pago, el único factor que está explicando el comportamiento de la industria farmacéutica.

Con base en esta hipótesis y partiendo de la premisa que son razones económicas, relacionadas con la potencial rentabilidad, las que influyen sobre las decisiones de la industria farmacéutica privada para invertir en la I+D de ciertos medicamentos, nos planteamos como objetivo de esta investigación desarrollar un modelo conceptual en el cual se identifiquen los factores asociados al comportamiento de la industria farmacéutica privada en cuanto a sus decisiones de inversión en I+D. El propósito, más allá de la definición del modelo, es avanzar en la identificación de esos factores, en el entendido de que su mejor conocimiento es primordial para el diseño de políticas públicas efectivas.

Para el desarrollo del modelo, nos basaremos en la teoría microeconómica relacionada con el comportamiento de los consumidores y de los productores y en la teoría de la maximización de los beneficios de los productores, haciendo énfasis en mercados con presencia de monopolios.

\section{Tres distinciones necesarias}

\section{Los mercados: interacción de dos agentes}

Los mercados están conformados por dos agentes económicos, el que compra o demanda y el que vende u ofrece. Entender el comportamiento de uno de los agentes económicos sin revisar el del otro y la forma cómo interactúan en el mer- cado resultaría un análisis incompleto. No es suficiente llegar a conclusiones sólo analizando el nivel de pago del consumidor, es necesario también conocer el nivel de costos de los productores en correspondencia con los niveles de pago del consumidor.

Tipo de medicamentos: vacunas, tratamientos curativos y tratamientos paliativos

Es un error realizar un mismo análisis para el mercado de vacunas, el de tratamientos curativos y el de tratamientos paliativos, ya que son productos diferentes, incluso aunque éstos atiendan una misma enfermedad. El comportamiento de la demanda y de la oferta para cada uno de ellos es distinto y está explicado por la naturaleza misma de cada bien, lo cual a su vez implica tener resultados diferentes en cada mercado.

Las confusiones en cuanto al análisis del comportamiento de la industria farmacéutica privada se originan porque se analizan indistintamente los incentivos para la investigación de una vacuna, una cura o un tratamiento paliativo. No es igual la demanda de la vacuna del SIDA, a la de la cura del SIDA o la del tratamiento del VIH/ SIDA, en primer lugar porque se trata de individuos distintos, los primeros son sanos y los otros ya contrajeron la enfermedad. En segundo lugar, cada uno de estos bienes satisface necesidades diferentes, uno es para prevenir la transmisión, el otro es para curar la enfermedad, y el último es para tratar la enfermedad.

Los factores que definen la demanda de cada uno de estos bienes no tienen igual peso en cuanto a su determinación, factores que por lo demás la industria farmacéutica es capaz no sólo de identificar sino de medir, ya que son los que les dan las orientaciones de cuál de esos mercados es potencialmente más rentable, información necesaria para la toma de decisiones de dónde invertir sus recursos de I+D.

\section{Tipo de enfermedades}

No podemos hacer igual análisis de las enfermedades transmisibles y las no transmisibles, e incluso dentro de las transmisibles hay diferencias, dependiendo de si el modo de transmisión es directo, indirecto o a través del aire, o de si cuentan con mecanismos, distintos a los medicamentos, para controlar la transmisión. Adicionalmente, existen enfermedades más letales que otras o que cuentan con tratamientos curativos o paliativos que sirven para atenuar dicha letalidad. Por lo tanto, para cada tipo de medicamento y de enfermedad debe realizarse un análisis particular del mercado. 


\section{La demanda de medicamentos}

\section{Algunas definiciones}

La demanda de un individuo se define como las cantidades de un bien o servicio que una persona está dispuesta a consumir a un precio dado y en un momento dado siempre que los otros factores (nivel de ingreso del consumidor, precio de los bienes relacionados, gustos y expectativas) permanezcan constantes 4 . La relación entre el precio y las cantidades demandadas nos permite conocer el tipo de función y su elasticidad, mientras que el comportamiento de los otros factores no define el tamaño de la demanda. Para efectos gráficos y de simplificación del análisis supondremos que la función de demanda de los medicamentos es una línea recta y por supuesto con pendiente negativa.

La elasticidad precio de la demanda es la variación porcentual de las cantidades demandadas cuando varía en $1 \%$ por ciento el precio. La demanda será más elástica cuando un incremento del $1 \%$ del precio implica una disminución mayor del $1 \%$ de las cantidades demandas. Las funciones de demanda son más inelásticas cuando un aumento del $1 \%$ del precio implica una disminución menor al $1 \%$ de las cantidades demandadas.

El que una función de demanda sea más elástica o inelástica depende de (1) lo necesario que es el producto para los demandantes, (2) la posibilidad de sustituirlo por otro bien y (3) cuánto se puede postergar la compra de ese bien. Los bienes muy necesarios, además muy difíciles de sustituir y cuya compra no puede ser postergada tienden a presentar una función de demanda más inelástica. La definición de elasticidad precio es relativa, una función de demanda es más o menos elástica con respecto a otra.

En la Figura la se muestran dos tipos de funciones de demanda, la demanda A es más inelástica que la B. Si el precio se ubicara en $\mathrm{P}_{0}$ para los dos tipos de demanda, las cantidades demandadas serían $\mathrm{qA}_{0} \mathrm{y} \mathrm{qB}_{0}$. Un incremento del precio a $\mathrm{P}_{1}$, implicará una disminución de las cantidades demandadas en menor proporción para el caso de la demanda A con respecto a la B. Para A, la disminución de las cantidades demandadas es $\left(\mathrm{qA}_{0}-\mathrm{qA}_{1}\right)$ y para $\mathrm{B}\left(\mathrm{qB}_{0}-\mathrm{qB}_{1}\right)$.

El tamaño de la demanda del individuo depende de factores distintos a la relación preciocantidad. Para el desarrollo de este modelo supondremos que en el caso de los medicamentos, los gustos, las expectativas de los individuos y el precio de los bienes relacionados no influyen y por lo tanto permanecen constantes, sólo ana- lizaremos el efecto que el ingreso tiene sobre el tamaño de la demanda del individuo.

Cuando el ingreso del individuo es menor, su demanda también es menor, y si su ingreso es mayor, también lo será la demanda. En la Figura 1b se observa la demanda A del individuo y para un precio $\mathrm{P}_{0}$ las cantidades demandadas serán $\mathrm{q}_{0}$. Si el ingreso aumentara, se reflejaría en un desplazamiento de la función de demanda paralelamente hacia la derecha para obtener una nueva función (demanda A') en la cual para el mismo precio $\mathrm{P}_{0}$ el individuo demandará mayor cantidad del bien, es decir $\mathrm{q}_{1}$.

Es importante mencionar que en el desarrollo de este modelo entenderemos por individuo no sólo a la persona enferma o potencialmente enferma, sino a quien efectivamente cubre los costos del medicamento, sea vacuna, cura o tratamiento paliativo: por lo tanto, se entiende, que en algunos casos, no necesariamente es la persona de manera directa quien paga el medicamento, sino que puede ser a través de intermediarios como los seguros o gobiernos.

Debemos distinguir entre la demanda del individuo y la demanda del mercado. La demanda del mercado es la agregación de todas las demandas individuales. Suponemos que todos los individuos tienen el mismo comportamiento e iguales condiciones, particularmente en cuanto a susceptibilidad y resistencia a las enfermedades y además cuentan con la misma información, por lo tanto, en las Figuras 1c, 1d y le observamos que la demanda del consumidor 1 es igual que la del consumidor 2 para el mismo producto, el individuo 1 demanda $\mathrm{q}_{1}$ cantidades y el individuo 2 $\mathrm{q}_{2}$, donde $\mathrm{q}_{1}=\mathrm{q}_{2}$. La demanda del mercado viene dada por la suma de $\mathrm{q}_{1}+\mathrm{q}_{2}$ para el mismo precio $\mathrm{P}_{0}$, por lo que la demanda del mercado es $\mathrm{Q}_{\mathrm{m}}$. En la medida que haya mayor número de individuos que demandan un bien, la demanda del mercado será mayor.

A continuación, estableceremos las características de la demanda para cada uno de los tipos de medicamentos incorporados en el modelo (vacunas, tratamientos curativos y paliativos). Estas características estarán dadas por (1) el tipo de función de demanda en cuanto a su elasticidad precio, (2) el tamaño de la demanda de cada individuo y (3) el tamaño de la demanda del mercado.

\section{La demanda de vacunas}

Las vacunas activan el sistema inmune para prevenir enfermedades transmisibles, por lo tanto, son demandadas por personas sanas. Dependiendo de la enfermedad, pudiésemos estar ante la presencia de demandas de vacunas más o me- 
Figura 1

Funciones de demanda del individuo y del mercado.

1a) Elasticidad precio de la demanda

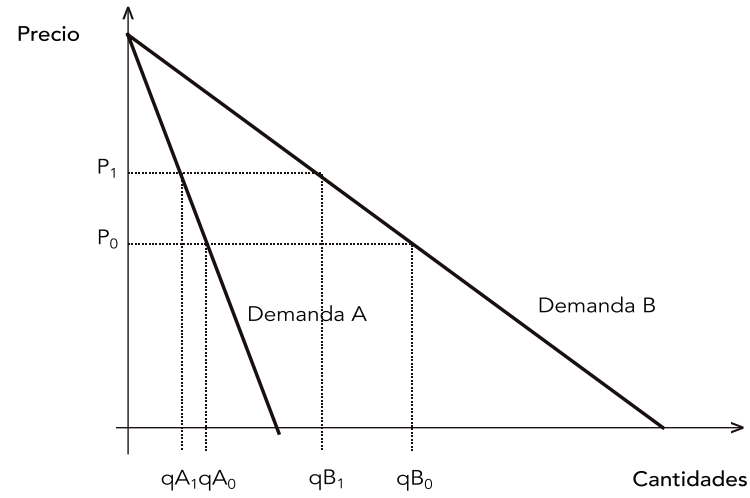

1c) Demanda del mercado: consumidor 1

1d) Demanda del mercado: consumidor 2
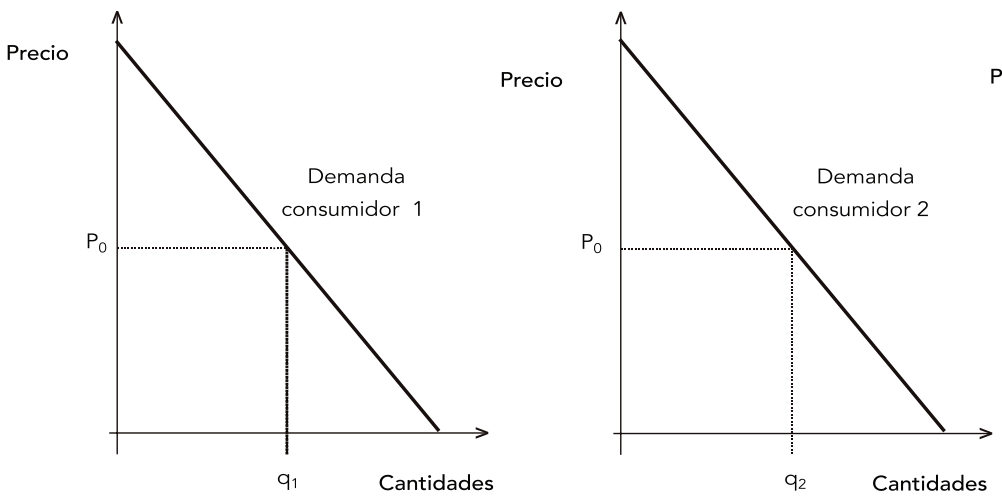

1b) Demanda del individuo

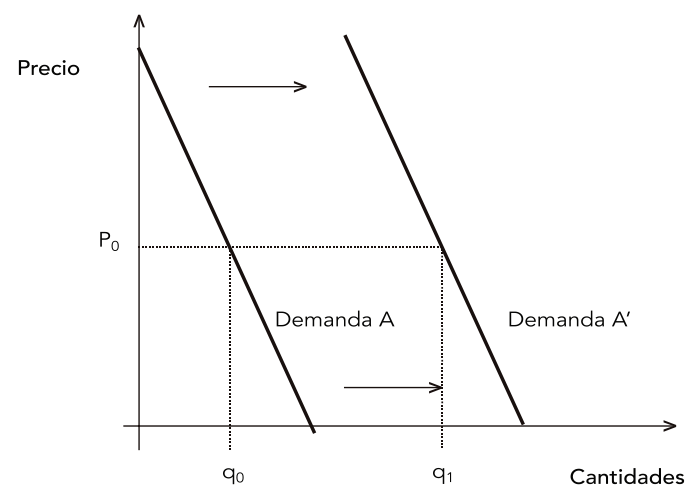

1e) Demanda del mercado

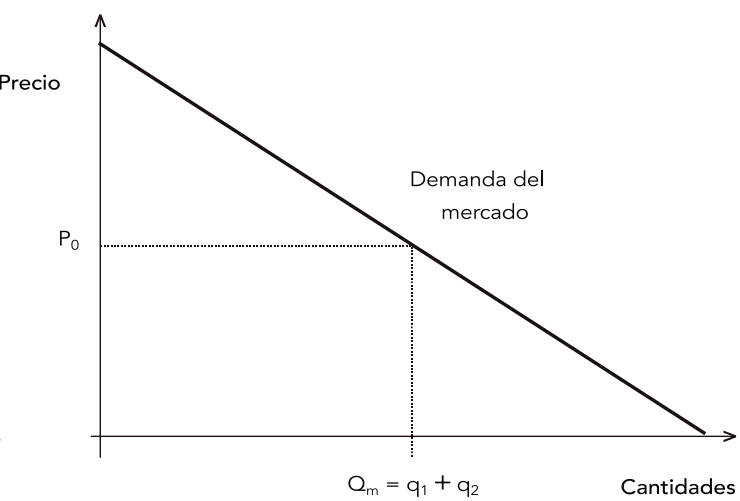

nos inelásticas, para lo cual, hemos identificado dos factores que consideramos están influyendo: (1) el modo de transmisión de la enfermedad y las posibilidades de controlar dicha transmisión mediante métodos distintos a la vacuna y (2) la letalidad de la enfermedad atenuada por la presencia de tratamientos curativos o paliativos.

Para determinar el tamaño de la demanda individual de la vacuna, el factor asociado que incorporaremos al modelo es el ingreso del individuo. Mientras que, el tamaño de la demanda del mercado de la vacuna será función del número de individuos dispuestos a comprarla. En la Tabla 1 se presentan los factores asociados a la demanda de vacunas.

\section{- Elasticidad precio de la demanda de vacunas}

a) Modo de transmisión de la enfermedad y posibilidades de controlar la transmisión

El modo de transmisión de una enfermedad (directo, indirecto o a través del aire) ${ }^{5}$ y las posibilidades de controlar la transmisión para prevenir la enfermedad nos permiten establecer qué tan necesaria es la vacuna y por lo tanto qué tan inelástica su demanda. En la medida que el modo de transmisión es a través del aire y es menos controlable su transmisión, la necesidad de contar con una vacuna es mayor y por consiguiente su demanda será más inelástica. Por el contrario, 
La demanda de vacunas.

\begin{tabular}{|c|c|c|}
\hline & Factores asociados & Características de la demanda \\
\hline \multirow[t]{2}{*}{ 1) Modo de transmisión de la enfermedad } & Métodos de control de la transmisión: & Elasticidad precio de la demanda \\
\hline & medidas de prevención distintas a las vacunas & \\
\hline \multirow[t]{2}{*}{ A través del aire } & No existen & Perfectamente inelástica \\
\hline & Existen & Muy inelástica \\
\hline \multirow[t]{2}{*}{ Directo } & No existen & Muy inelástica \\
\hline & Existen & Moderadamente inelástica \\
\hline \multirow[t]{2}{*}{ Indirecto } & No existen & Muy inelástica \\
\hline & Existen & Moderadamente inelástica \\
\hline 2) Letalidad & Presencia de tratamiento curativo o paliativo & \\
\hline \multirow[t]{4}{*}{ Alta } & Existe tratamiento curativo & Moderadamente inelástica \\
\hline & Existe tratamiento paliativo & Moderadamente inelástica \\
\hline & No existe tratamiento curativo & Perfectamente inelástica \\
\hline & No existe tratamiento paliativo & Perfectamente inelástica \\
\hline \multirow[t]{4}{*}{ Baja } & Existe tratamiento curativo & Moderadamente inelástica \\
\hline & Existe tratamiento paliativo & Moderadamente inelástica \\
\hline & No existe tratamiento curativo & Muy inelástica \\
\hline & No existe tratamiento paliativo & Muy inelástica \\
\hline \multirow[t]{2}{*}{ Ninguna } & & Elástica \\
\hline & & Tamaño de la demanda del individuo \\
\hline \multirow[t]{3}{*}{ Nivel de ingreso de cada individuo } & Alto & Mayor demanda \\
\hline & Bajo & Menor demanda \\
\hline & & Tamaño de la demanda del mercado \\
\hline \multirow[t]{3}{*}{ Número de demandantes en el mercado } & Aumenta & La demanda aumenta \\
\hline & Disminuye & La demanda disminuye \\
\hline & Constante & La demanda es constante \\
\hline
\end{tabular}

cuando más posibilidades existen de controlar la transmisión, las demandas tenderán a ser menos inelásticas. Hemos clasificado 4 grados de inelasticidad de la demanda que van desde la más a la menos inelástica en el siguiente orden: perfectamente inelástica, muy inelástica, moderadamente inelástica y elástica.

Las demandas de la vacuna para el sarampión o la gripe aviar que se transmiten de modo directo y cuyas posibilidades de controlar la transmisión son muy reducidas tienden a ser muy inelásticas, si las comparamos con la demanda de la vacuna del SIDA, que por tratarse de una enfermedad que aunque también se transmite de manera directa por contacto sexual o indirecta ${ }^{5}$, tendría, de acuerdo con el modelo, una función de demanda moderadamente inelástica en la medida en que existen mecanismos de control de la transmisión.

La demanda de la vacuna de la viruela (en su momento) podría caracterizarse como perfectamente inelástica, debido al modo de transmisión aerógeno sin posibilidades de control, de igual manera que la demanda de la vacuna de la gripe. La malaria que se transmite de modo indirecto, a través de un vector y con posibilidades de controlar la transmisión, ya sea con medidas preventivas comunitarias o personales, tendrá funciones de demandas de vacuna moderadamente inelásticas.

\section{b) Letalidad de la enfermedad}

Otro factor importante para definir la elasticidad precio de la demanda de la vacuna es la letalidad de la enfermedad. Cuando las enfermedades son más letales, independientemente de su modo de transmisión y de la posibilidad de controlarla, la necesidad de contar con una vacuna para prevenir la enfermedad es mayor. El análisis de la letalidad debe estar acompañado con variables que indiquen la presencia o no de tratamientos curativos o paliativos de dicha enfermedad, ya que éstos podrían atenuar la letalidad.

La demanda de la vacuna del sarampión que habíamos definido muy inelástica por su modo 
de transmisión, también será muy inelástica por su baja letalidad (de 3\% a 5\% en países en desarrollo 5) aunque no cuente con tratamientos específicos. Sin embargo, la demanda de la vacuna de la gripe aviar, que habíamos clasificado como muy inelástica pasará a ser perfectamente inelástica, debido a su alta letalidad (en el brote actual ha muerto más de la mitad de las personas infectadas por el virus 6 ) y ausencia de tratamientos específicos.

En cuanto a la viruela, la demanda de su vacuna seguirá siendo perfectamente inelástica, debido a su alta letalidad (entre el 20\% y $40 \%$ para el caso de la variola mayor-clásica 5) y ausencia de tratamiento. A diferencia de la influenza, que aunque no cuenta con tratamientos efectivos, sólo con algunos que aplicándose de manera oportuna alivian el malestar, es de baja letalidad y pasará a ser una demanda muy inelástica. La malaria, cuya tasa de letalidad puede ser entre el $10 \%$ y $40 \%$, al contar con tratamientos específicos como la cloroquina 5 mantendrá una función de la demanda de vacuna moderadamente inelástica.

Lo que queremos plantear es que no todas las demandas de vacunas tienen la misma elasticidad, existen unas perfectamente inelásticas, otras muy inelásticas y otras moderadamente inelásticas. Esto es muy importante para entender el comportamiento de la industria farmacéutica, ya que los beneficios de la industria serán potencialmente mayores en la medida que las demandas sean más inelásticas.

\section{- Demanda de vacuna del individuo}

La demanda de la vacuna será mayor o menor para cada precio dependiendo del nivel de ingreso del consumidor. Cuando éste es alto, la demanda también será alta y estará más alejada del origen de coordenadas, mientras que con un nivel de ingreso menor la demanda será menor y más cerca del origen de coordenadas (Figura 1b). Esto explica por qué la demanda individual de la vacuna de la malaria, la leihsmaniasis, o la enfermedad del sueño es pequeña y muy cercana al origen de coordenadas, ya que dichas enfermedades se manifiestan en países en desarrollo y sus demandantes cuentan con bajos ingresos.

\section{- Demanda de vacuna del mercado}

Cuando el número de personas que demandan una vacuna es mayor, la demanda del mercado será mayor, ubicándose cada vez más lejos del origen de coordenadas. Para la industria farmacéutica es más atractivo el mercado que tiene mayor número de demandantes y por lo tanto mayor demanda de mercado.

Para el caso de las vacunas, y no necesariamente para el otro tipo de medicamentos, el número de personas que demandan este bien puede ser muy elevado al inicio, luego permanecer constante hasta incluso ir disminuyendo en el tiempo. Cuando, por ejemplo, se descubre la vacuna se inicia con la inmunización de la mayoría de la población y puede llegar un momento en el que si se erradica le enfermedad no haya demanda de la vacuna como sucedió con la viruela. Esta es una razón por la cual los mercados de vacunas, en su mayoría, no son muy atractivos para la industria farmacéutica privada, al menos que la demanda sea perfectamente inelástica, debido al alto factor de riesgo de transmisión y la alta letalidad.

\section{La demanda de tratamientos curativos}

\section{- Elasticidad precio de la demanda de tratamientos curativos}

Los tratamientos curativos son demandados por personas que ya están enfermas y que necesitan una cura. Puesto que la enfermedad ya se adquirió, el factor modo de transmisión y control de dicha transmisión ya no es importante. La elasticidad de las funciones de demanda de los tratamientos curativos estará determinada por el nivel de letalidad de la enfermedad.

En la Tabla 2 observamos que en la medida que la enfermedad es más letal y no existe la posibilidad de tratamientos paliativos, la demanda de la cura será perfectamente inelástica, como por ejemplo, el cáncer. En cambio, si la enfermedad es muy letal, pero existe un tratamiento paliativo, la demanda de la cura pasaría a ser muy inelástica en lugar de perfectamente inelástica, ya que existe la posibilidad de seguir viviendo con la enfermedad aplicándose el tratamiento. Esto sucede con, por ejemplo, la demanda de la cura de la diabetes, que aunque es letal, la presencia de tratamientos paliativos (la insulina) hace que la demanda de la cura pase a ser de perfectamente elástica a muy inelástica.

Cuando la enfermedad es de letalidad baja y existe un tratamiento paliativo, la demanda de la cura de esta enfermedad tiende a ser moderadamente inelástica. Por último, cuando la enfermedad no es letal, la demanda de la cura tiende a ser elástica.

\section{- Demanda de tratamientos curativos del individuo}

El factor asociado al tamaño de la demanda es el ingreso del individuo, y a mayor ingreso, mayor 
La demanda de tratamiento curativo.

\begin{tabular}{|c|c|c|}
\hline & Factores asociados & Características de la demanda \\
\hline 1) Letalidad & Presencia de tratamiento curativo o paliativo & Elasticidad precio de la demanda \\
\hline \multirow[t]{2}{*}{ Alta } & Existe tratamiento paliativo & Muy inelástica \\
\hline & No existe tratamiento paliativo & Perfectamente inelástica \\
\hline \multirow[t]{2}{*}{ Baja } & Existe tratamiento paliativo & Moderadamente inelástica \\
\hline & No existe tratamiento paliativo & Muy inelástica \\
\hline \multirow[t]{2}{*}{ Ninguna } & & Elástica \\
\hline & & Tamaño de la demanda del individuo \\
\hline \multirow[t]{3}{*}{ Nivel de ingreso de cada individuo } & Alto & Mayor demanda \\
\hline & Bajo & Menor demanda \\
\hline & & Tamaño de la demanda del mercado \\
\hline \multirow[t]{3}{*}{ Número de demandantes en el mercado } & Aumenta & La demanda aumenta \\
\hline & Disminuye & La demanda disminuye \\
\hline & Constante & La demanda es constante \\
\hline
\end{tabular}

será la demanda dado un precio del tratamiento $\mathrm{y}$ viceversa.

\section{- Demanda de mercado de tratamientos curativos}

En la medida que más personas desarrollen alguna enfermedad y demanden una cura, en esa medida la demanda del mercado para el tratamiento curativo será mayor y viceversa.

\section{La demanda de tratamientos paliativos}

\section{- Elasticidad precio de la demanda de tratamientos paliativos}

Los tratamientos paliativos son demandados por personas que presentan una enfermedad que por lo general no tiene cura, para mitigar su violencia y refrenar su rapidez. Tomando en cuenta los mismos supuestos que para el caso de los tratamientos curativos, la elasticidad precio de la demanda para el caso de tratamientos paliativos dependerá del nivel de letalidad de la enfermedad. En la Tabla 3 se observa que cuando la enfermedad es muy letal y no existe una cura para dicha enfermedad, la demanda del tratamiento paliativo tiende a ser perfectamente inelástica, ya que es la única opción que la persona tiene para sobrevivir aunque sea enferma, ejemplo de estas enfermedades la diabetes con el tratamiento de insulina.

En la medida que la enfermedad es menos letal, la demanda del tratamiento paliativo pasará a ser muy inelástica. Cuando la enfermedad no es letal, cualquier tratamiento paliativo tendrá demandas elásticas.

\section{- Demanda de tratamiento paliativo del individuo}

A mayor ingreso, mayor será la demanda dado un precio del tratamiento paliativo y viceversa.

\section{- Demanda de mercado del tratamiento paliativo}

Cuando el número de personas enfermas es mayor, también será mayor la demanda del mercado de tratamientos paliativos. Adicionalmente, en la medida en que el tratamiento paliativo permite que la persona pueda vivir con la enfermedad un mayor número de años, el número de personas enfermas que anualmente requerirán el tratamiento será cada vez mayor, ya que en el mercado concurrirán las personas ya enfermas, que adquieren el tratamiento y que viven mayor número de años, más los nuevos enfermos.

\section{La oferta de medicamentos}

La industria farmacéutica, en la mayoría de los casos se comporta como un monopolio. La posibilidad que les otorga el Acuerdo de Derechos de Propiedad Intelectual, suscrito en el marco de la Organización Mundial del Comercio a la industria farmacéutica por la invención de un producto, les garantiza la exclusividad para la producción y comercialización de dicho bien. Se crea 
La demanda de tratamiento paliativo.

\begin{tabular}{|c|c|c|}
\hline & Factores asociados & Características de la demanda \\
\hline 1) Letalidad & Presencia de tratamiento curativo o paliativo & Elasticidad precio de la demanda \\
\hline \multirow[t]{2}{*}{ Alta } & Existe tratamiento curativo & No aplica, la demanda se dirige a la cura \\
\hline & No existe tratamiento curativo & Perfectamente inelástica \\
\hline \multirow[t]{2}{*}{ Baja } & Existe tratamiento curativo & No aplica, la demanda se dirige a la cura \\
\hline & No existe tratamiento curativo & Muy inelástica \\
\hline \multirow[t]{2}{*}{ Ninguna } & & Elástica \\
\hline & & Tamaño de la demanda del individuo \\
\hline \multirow[t]{3}{*}{ Nivel de ingreso de cada individuo } & Alto & Mayor demanda \\
\hline & Bajo & Menor demanda \\
\hline & & Tamaño de la demanda del mercado \\
\hline \multirow[t]{3}{*}{ Número de demandantes en el mercado } & Aumenta & La demanda aumenta \\
\hline & Disminuye & La demanda disminuye \\
\hline & Constante & La demanda es constante \\
\hline
\end{tabular}

un monopolio legal que concede a la industria un poder de mercado al fijar precios y cantidades. Para efectos de simplificación del análisis, supondremos que la estructura de costos de la industria farmacéutica se comporta de manera tal que tanto los costos marginales (CMg - lo que cuesta producir una unidad adicional del bien) como los costos medios totales (CMeT) son iguales y constantes independientemente de las cantidades producidas.

Incorporaremos el concepto de los costos de oportunidad de la industria farmacéutica, es decir, aquellos en los cuales se incurre cuando se deja de realizar una actividad por realizar otra, éstos forman parte de los costos totales y por lo tanto tienen implicaciones sobre la rentabilidad de la empresa y son imprescindibles para entender el comportamiento de las empresas al momento de evaluar opciones de inversión.

\section{Maximización de beneficios de la industria farmacéutica privada}

Las industrias maximizan su beneficio cuando sus ingresos marginales (IMg) son iguales a sus costos marginales, en la Figura 2 se trata del punto A en el que se producirían $\mathrm{q}_{0}$ del bien a un precio de $\mathrm{P}_{0}$. Por su condición de monopolio, podría producir a un nivel de precio en el que la diferencia del ingreso total menos el costo total sea máxima, es decir, donde sus beneficios sean mayores. Para ello, en el nivel de producción $\mathrm{q}_{0}$, en lugar de fijar el precio en $\mathrm{P}_{0}$, lo proyectará hasta $\mathrm{P}_{1}$, ubicándose en el punto $\mathrm{B}$ de la función de demanda. Sus ingresos totales vienen dados por el área del rectángulo $0 \mathrm{q}_{0} \mathrm{BP}_{1}$, los costos totales están representados por el área del rectángulo $0 \mathrm{q}_{0} \mathrm{AP}_{0}$ y los beneficios totales son el área del rectángulo $\mathrm{P}_{0} \mathrm{ABP}_{1}$.

Si se incrementara la demanda del mercado debido, por ejemplo, a un mayor número de demandantes, se verá reflejado en la gráfica por un desplazamiento paralelo de la función de demanda hacia la derecha, como fue mostrado en la Figura 1b y en este caso, dada una misma función de costos medios totales y marginales, las cantidades y el precio de equilibrio serán mayores y por consiguiente los beneficios también serán mayores.

Si por ejemplo, los costos del productor aumentaran, las funciones de CMg y CMeT, que supusimos son iguales y constantes, se desplazarán hacia arriba. La consecuencia, dada una misma función de demanda, es una disminución del beneficio del productor. Más aún, si el incremento de los costos es muy alto, puede suceder que las funciones de costos queden por encima de la de demanda sin posibilidad de un punto de encuentro entre el IMg y el CMg (condición de la maximización de beneficio del productor). En ese caso, el productor no tendrá interés de invertir en este mercado.

\section{Incentivo de la industria farmacéutica privada para la I+D}

Con base en el modelo desarrollado, explicaremos cómo se comporta la industria farmacéutica en cuanto a su inversión en I+D. Para lo cual ilustraremos tres casos. 


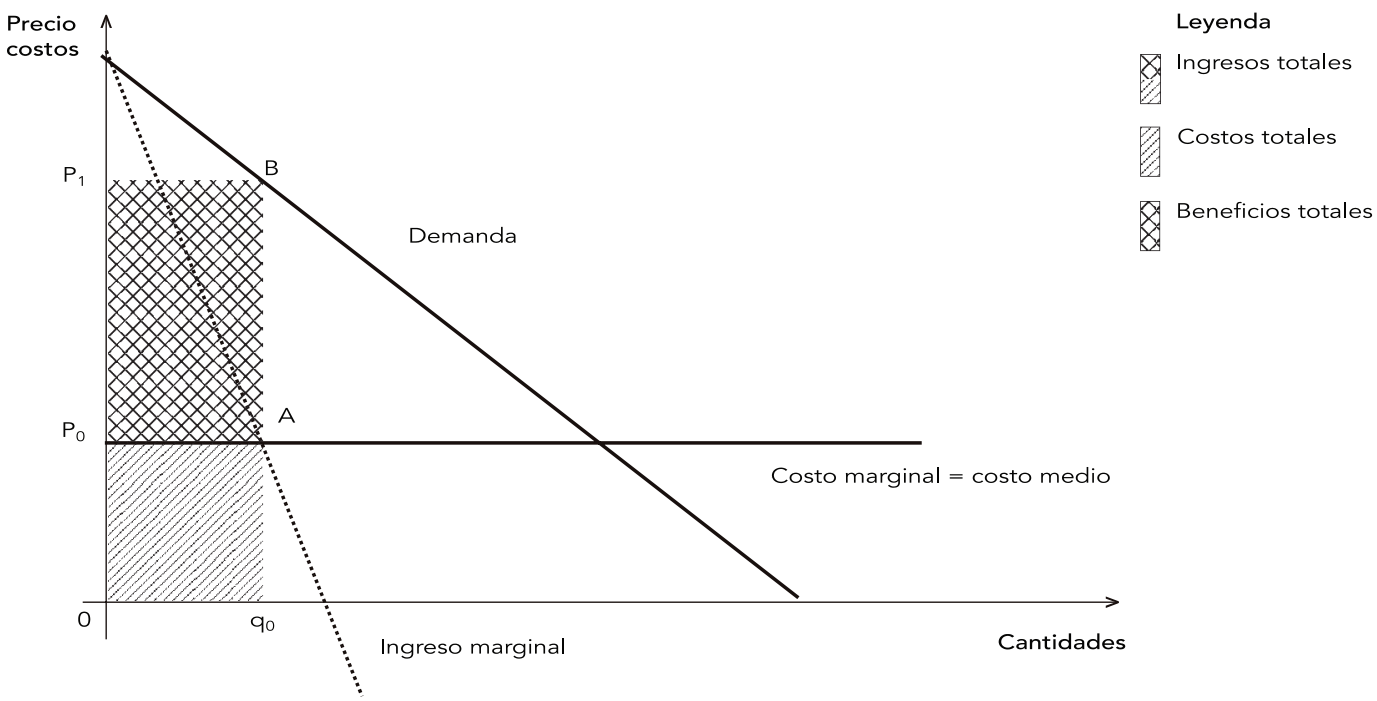

\section{Caso 1: la cura de la diabetes}

La Figura 3a muestra el mercado del tratamiento paliativo de la diabetes y el 3B el de la cura de la misma enfermedad. En el primer caso observamos que la función de demanda (D1) es casi perfectamente inelástica, debido a que se trata de una enfermedad letal e incurable. Además es una demanda de mercado relativamente grande, esto por dos razones, en primer lugar porque se trata de individuos que habitan en países con niveles de ingresos medianos y altos, y en segundo lugar porque son muchos los individuos que demandan el tratamiento, actualmente hay 246 millones de personas diabéticas en el mundo (aunque no todos demandan el bien). El monopolista maximizará su beneficio donde su CMg es igual al ingreso marginal correspondiente a la demanda 1 (IMg1), esto es en el punto A, produciendo $\mathrm{q}_{0}$ cantidades del tratamiento a un precio proyectado de $\mathrm{P}_{1}$.

La demanda de la cura de la diabetes, la graficamos de acuerdo con el modelo como muy inelástica, debido a que cuenta con un tratamiento paliativo. En cuanto a los costos, $\left(\mathrm{CMeT}_{0}\right.$ $=\mathrm{CMg}_{0}$ ) éstos son muy elevados y se encuentran por encima de la función de demanda debido a que incluyen, además de los de producción y los de la recuperación de la $\mathrm{I}+\mathrm{D}$, los costos de oportunidad de dejar de ganar en el mercado del tratamiento paliativo si existiese una cura de la enfermedad. No existe un punto de maximización de beneficio, donde el monopolista esté dispuesto a invertir en ese mercado.

Más aún, con el tiempo, la demanda del tratamiento paliativo irá aumentando y desplazándose de manera paralela hasta $\mathrm{D}_{2}$, esto como consecuencia de sumar los nuevos enfermos que requieren el tratamiento. Dada esa nueva demanda $\mathrm{D}_{2}$, en el mercado del tratamiento paliativo, el monopolista se ubicará en el punto $\mathrm{B}$, producirá $\mathrm{q}_{1}$ a un precio proyectado de $\mathrm{P}_{1}$. Su beneficio que antes era el área del rectángulo $\mathrm{P}_{0} \mathrm{ACP}_{1}$ ahora es mayor y viene dado por el área $\mathrm{P}_{0} \mathrm{BDP}_{1}$. Esta demanda estará en permanente aumento toda vez que el número de nuevas personas enfermas anualmente (7 millones) sea mayor que el número de personas que fallecen a causa de esta enfermedad (3 millones de personas anualmente).

El permanente incremento del beneficio de la empresa en el mercado del tratamiento paliativo se corresponde con un incremento del costo de oportunidad en el mercado de la cura de la diabetes, lo cual se muestra en la Figura 3 b como un desplazamiento paralelo de la funciones de costos (medios totales y marginales) hacia arriba hasta $\mathrm{CMeT}_{1}=\mathrm{CMg}_{1}$, haciendo que la brecha entre la función de demanda y los costos de la em- 
Figura 3

Mercados del tratamiento de la diabetes.

3a) Tratamiento paliativo

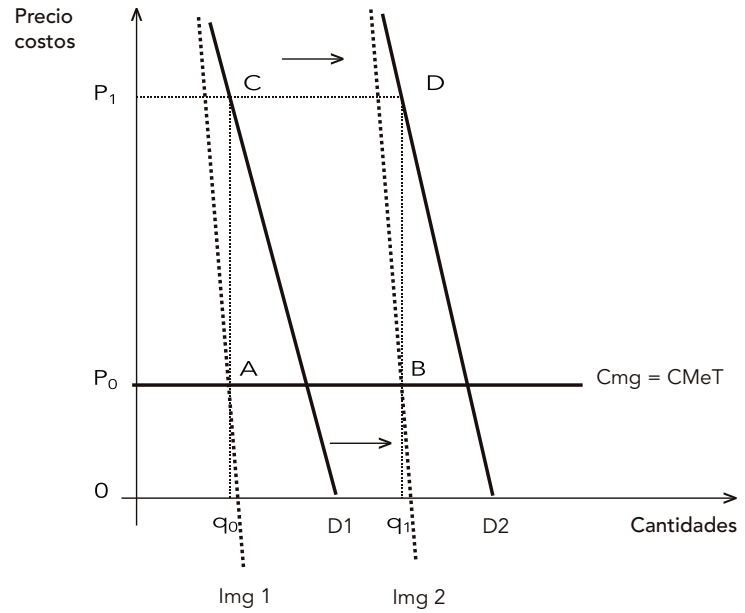

3b) Tratamiento curativo

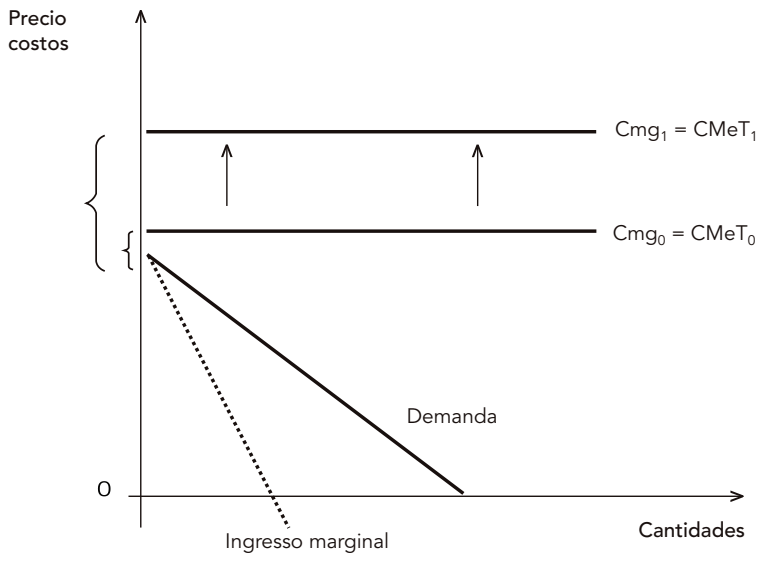

presa sea cada vez mayor. Esta brecha aumentará cada vez que aumente la demanda del tratamiento paliativo de la diabetes.

Esto explica por qué aunque el nivel de ingreso de los demandantes de la cura de la diabetes es alto y por lo tanto pudiese incentivar a la industria para que invierta en I+D de la cura de esta enfermedad, no existe por el contrario tal incentivo, sino más bien se presenta como un desincentivo para la industria.

Incluso, si la empresa no se comportara como un monopolio, y por el contrario concurren distintas empresas, el efecto del costo de oportunidad reflejado en el mercado de la cura de la diabetes se seguirá manifestando aunque con menor nivel, ya que el incremento de la demanda del tratamiento paliativo deberá repartirse entre todos los oferentes, por lo que el costo de oportunidad será menor para cada empresa, pero seguirá presente.

\section{Caso 2: la vacuna para la malaria}

En la Figura 4 se muestra el mercado de la vacuna de la malaria, el cual se caracteriza por una demanda moderadamente inelástica, debido a que - de acuerdo con el modelo - se trata de una enfermedad que se transmite de modo indirecto y con posibilidades de controlar dicha transmisión, adicionalmente cuenta con tratamientos curativos. El tamaño de la demanda es relativamente pequeño por dos razones, en primer lugar porque la enfermedad se manifiesta en países de bajos ingresos, lo que hace que la demanda de cada individuo sea pequeña por su baja capacidad adquisitiva, y en segundo lugar, la demanda del mercado es relativamente pequeña porque la enfermedad sólo se manifiesta en las regiones tropicales.

Los costos de la empresa, incorporando los costos de I+D, son relativamente altos si los comparamos con la demanda, por lo que no existe un nivel donde la empresa pueda maximizar sus beneficios y no tiene ningún tipo de incentivo para invertir en la I+D de esta vacuna. Esto explica, desde el punto de vista de los mercados y de los incentivos económicos por qué la industria farmacéutica no invierte recursos para investigar en enfermedades como la malaria, el chagas, la enfermedad del sueño, es decir, las enfermedades que se han denominado "descuidadas u olvidadas".

\section{Caso 3: la vacuna para la gripe aviar}

La gripe aviar es una enfermedad que se trasmite de modo directo, además de ser una enfermedad con pocas posibilidades para controlar el contagio, es muy letal y no cuenta con tratamiento curativo o paliativo, por lo tanto podríamos decir, 
de acuerdo con el modelo, que la demanda de la vacuna de esta enfermedad es casi perfectamente inelástica. Suponiendo que el tamaño de la demanda de mercado es pequeño, debido a que sólo se ha manifestado en países en desarrollo, una demanda de este tipo, dada una estructura de costos de la industria, permitirá que siempre se logre un punto de maximización de beneficios de la industria, es decir, un punto donde el IMg sea igual al CMg (Figura 5).

No importa lo altos que sean los costos marginales, siempre se podrá maximizar el beneficio, ya que se trata de un bien que los individuos están dispuestos a demandar independientemente del precio, éste puede ser tan alto como lo establezca el mercado o el monopolio, de acuerdo con la estructura de costos de la empresa. Esto explica por qué en cuatro años desde que se diagnosticó el primer caso de la gripe aviar, y a pesar de que sólo se ha manifestado en países en desarrollo, los recursos orientados a la I+D por parte de la industria para desarrollar una vacuna han permitido que, en tan poco tiempo, se haya avanzado en una posible vacuna para combatirla. Debemos mencionar que dadas las características de rápido contagio de la enfermedad, y debido a la globalización, la gripe aviar, adicionalmente, mantiene en alto riesgo a los países de altos ingresos.

\section{Conclusiones}

La industria farmacéutica, actuando como un monopolio, invertirá en aquellos mercados cuyas funciones de demanda sean más inelásticas y/o de mayor tamaño. No tendrá incentivos para invertir en los mercados donde sus costos marginales superan los ingresos marginales.

Existen mercados que aunque sus consumidores cuenten con elevada capacidad de pago, no necesariamente son atractivos para que la industria farmacéutica invierta en I+D y en algunos casos más bien existe el efecto contrario en hallar la cura o la vacuna de ciertas enfermedades dado los costos de oportunidad que esto le implica.

Las políticas orientadas a incentivar a la industria privada para que investigue y desarrolle algunos medicamentos como son las políticas de "arrastre" en la que se trata de garantizar a la empresa un mercado a través de un compromiso de compra del medicamento una vez desarrollado y de "empuje" basada en subsidios a la investigación 7, en algunos casos no serán efectivas. Cuando el costo de oportunidad de invertir en el desarrollo de una vacuna o cura es cada vez mayor, el esfuerzo en términos económicos de un subsidio que permita desplazar las

\section{Figura 4}

Mercado de la vacuna de la malaria.

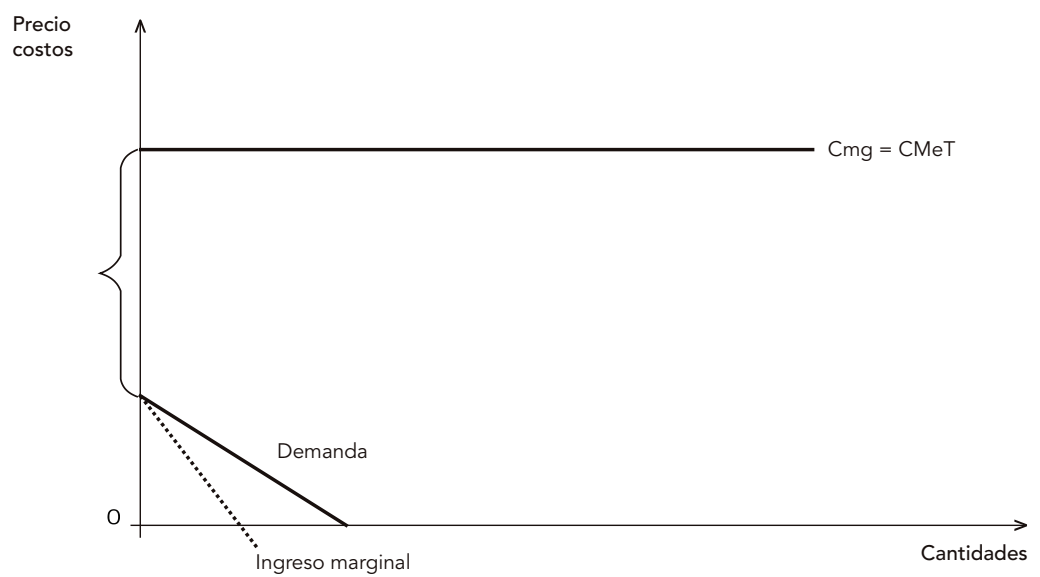

Figura 5

Mercado de la vacuna de la gripe aviar.

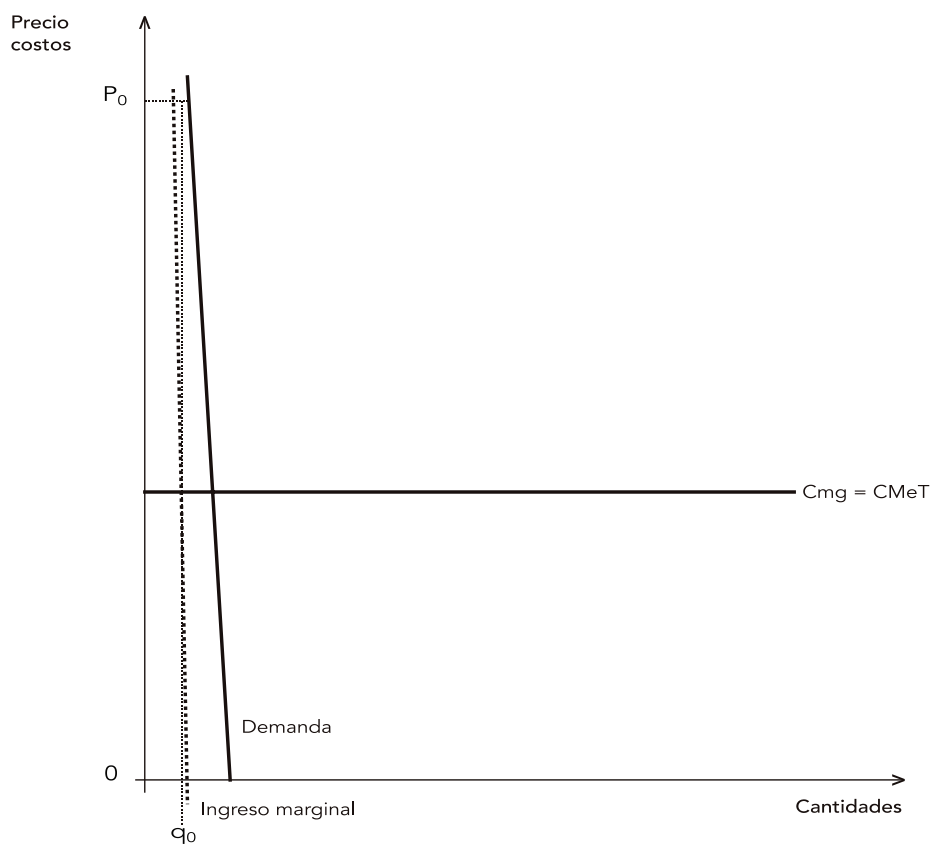


funciones de costos de la empresa hacia abajo hasta un nivel que se encuentren con la demanda, es muy elevado, debería cubrir la rentabilidad que la industria deja de obtener a largo plazo en el mercado del tratamiento paliativo más el costo de I+D. Por su parte, la política de "arrastre", aunque busque garantizar la demanda de la vacuna o de la cura, tiene sus límites y, en algunos casos, con el tiempo el límite es cada vez menor (por ejemplo, cuando se erradique la enfermedad).

Este tipo de políticas podrían resultar efectivas para mercados que no tienen implícito los costos de oportunidad y cuyas demandas de mercado son pequeñas, debido a la baja capacidad de pago de los consumidores y al bajo número de demandantes, como es por ejemplo el caso de las enfermedades olvidadas. En todo caso, el que estas políticas puedan ser efectivas dependerá de la brecha que existe entre los costos de la empresa y la demanda.
Por lo mencionado anteriormente, las políticas que tengan como objetivo promover el desarrollo de medicamentos en este tipo de enfermedades, deben necesariamente orientarse a un fortalecimiento de las capacidades, recursos e infraestructura para la realización de investigación básica y aplicada por parte de las instituciones públicas o privadas sin fines de lucro. Es a través del fortalecimiento de las instituciones públicas dedicadas a la investigación como se podrá avanzar en el desarrollo de estos medicamentos que no son de interés de la industria privada.

Las políticas de regulación de los monopolios, a través de la fijación de precios máximos y cantidades mínimas, contribuirían no sólo a garantizar un mayor acceso a los medicamentos, sino a disminuir el costo de oportunidad de la industria farmacéutica, en aquellos casos de enfermedades con tratamientos de largo plazo, y por lo tanto a disminuir las brechas entre la demanda y los costos en el mercado de las vacunas y las curas asociadas a estas enfermedades.

\section{Resumen}

En el trabajo se desarrolló un modelo con los factores que influyen sobre la decisión de $I+D$ de la industria farmacéutica. Los factores son el riesgo de contagio y la posibilidad de controlarlo; la letalidad y la presencia de curas o tratamientos; el ingreso; el número de personas que demandan y los costos de oportunidad de la empresa. Las empresas invertirán en mercados con demandas más inelásticas (enfermedades muy contagiosas sin posibilidad de controlar la transmisión y/o muy letales sin curas o tratamientos) y/o donde los demandantes tienen elevados ingresos y/o son numerosos. No invertirá en mercados donde sus costos marginales son superiores a sus ingresos marginales, particularmente cuando sus costos incrementan permanentemente como consecuencia del aumento del costo de oportunidad generado por lo que dejaría de ganar en otros mercados. Para estos casos, las políticas de subsidio de la I+D no son efectivas, por lo tanto, éstas deben orientarse al fortalecimiento de las investigaciones, tanto básica como aplicada, realizadas por las instituciones públicas o sin fines de lucro.

Industria Farmacéutica; Políticas de Salud; Preparaciones Farmacéuticas; Motivación 


\section{Referencias}

1. Médicos Sin Fronteras. Desequilíbrio fatal: a crise em pesquisa e desenvolvimento de drogas para doenças negligenciadas. Geneva: Médicos Sin Fronteras; 1999.

2. Organización Mundial de la Salud. Salud pública: innovación y derechos de propiedad intelectual. Ginebra: Organización Mundial de la Salud; 2006. (Informe de la Comisión de Derechos de Propiedad Intelectual, Innovación y Salud Pública).

3. International Diabetes Federation. Diabetes atlas. 3rd Ed. http://www.eatlas.idf.org/webdata/docs/ background_opening_pc.pdf (accedido el 19/ Mar/2007)

4. Nicholson W. Microeconomía intermedia. 8ạ Ed. Madrid: McGraw-Hill; 2001.
5. Organización Panamericana de la Salud. El control de las enfermedades transmisibles. 17ạ Ed. Washington DC: Organización Panamericana de la Salud; 2001.

6. World Health Organization. Avian influenza: frequently asked questions. Wkly Epidemiol Rec 2005; 80:377-84.

7. Arhin-Tenkorang D, Conceição P. Beyond communicable disease control: health in the age of globalization. In: Kaul I, Conceição P, Le Goulven K, Mendoza RU, editors. Providing global public goods: managing globalization. Oxford: Oxford University Press; 2003. p. 484-515.

Recibido el 30/Abr/2007

Versión final presentada el 11/Dic/2008

Aprobado el 06/Mar/2008 